\title{
Safety matters
}

\author{
Two Q\&As in this issue consider aspects of chemical safety in industry and academia. And when it comes to the \\ latter, a Review Article concludes that much more research is required to better understand - and improve - \\ safety in academic laboratories.
}

T he April 2019 issue of Nature Chemistry marked the journal's tenth anniversary and to celebrate the occasion we asked a diverse group of chemists to tell us what they thought the most exciting, interesting or challenging developments related to their areas of expertise would be in the coming years. The vast majority of the responses, which we collected together in a Feature article (Nat Chem. 11, 286-294; 2019), focused on specific research topics - such as catalysis, artificial intelligence and sustainability - but Marc Reid from the University of Strathclyde took a somewhat different approach. He noted that the most important aspect of future developments in chemistry is that they should be accomplished safely and he concluded with a question: how can chemists better understand their failings when it comes to laboratory safety?

This question is not a simple one and there is no easy answer; the scale of the challenge is highlighted in a Review Article in this issue. Two researchers at the University of Windsor, Canada — Dana Ménard (a clinical psychologist) and John Trant (a synthetic organic chemist) - teamed up to examine various aspects of laboratory safety in academic settings, including the types of accidents that occur, how often they happen, the factors that contribute to them and what research has been done on the subject. Despite several major incidents during the past decade in which researchers have suffered serious injuries or lost their lives, Ménard and Trant note that the study of safety in academic laboratories is not very well developed and there is a significant lack of data when it comes to the attitudes and behaviour of researchers. The ultimate conclusion of the Review Article, that the academic discipline does not prioritize safety, is damning.

Researchers who have worked in academic chemistry laboratories will typically be aware of safety-related incidents that have occurred in their own research group, or perhaps in other groups in a university department if the incident was significant enough to attract wider attention. Barring major injuries or damage to infrastructure, however, knowledge of such events is not always shared or documented in a systematic fashion and simply becomes anecdote spread by word of mouth. As Ménard and Trant point out, the lack of a comprehensive dataset of accidents and near-misses in academic laboratories makes the study of safety incredibly difficult.

Unsurprisingly, researchers are broadly positive about the general idea of safety. However, Ménard and Trant highlight that there have been no investigations as to whether there is a correlation between attitudes towards safety and the implementation of safe laboratory practice. And there does seem to be a disconnect; studies of safety practices suggest that many researchers do not complete the necessary assessments before doing an experiment. The results of one particular study revealed that researchers in an academic environment were much less likely to assess risks than their counterparts in industrial or government laboratories.

Also discussed in the Review Article is another aspect of laboratory safety that can undermine efforts to improve the culture across academia: the tendency to focus on who is to blame - typically the individual involved in the accident is quickly singled out - rather than using such incidents as a learning opportunity in an effort to reduce the likelihood of further such events. The cause of chemical accidents are often complex and can be the result of a host of different factors, so it is important that questions are asked that can lead to a better understanding of what resulted in the unsafe situation in the first place. There are lessons to be learned in every safety-related incident, even minor ones, but if the person affected is concerned about being judged, then cases are more likely to go unreported.

One area that has garnered a little more research attention is the safety training received by undergraduate students. As Ménard and Trant note, it is important to get this training right, because if this is perceived to be an institutionally mandated hassle, then that can set the tone for an individual's negative attitude towards safety as they progress through their career.

Motivated by Reid's contribution to our ten-year-anniversary Feature article, we decided to follow-up with him in a Q\&A piece that is published in this issue. Reid is not only a research academic but also a director of a safety-focused company Pre-Site Safety. He explains both his professional and personal motivations for establishing Pre-Site Safety and highlights how his passion for safety awareness has influenced his research group. A positive attitude towards safety is fostered in Reid's group with safety recaps each week in which there is open discussion of any accidents or near-misses. He also discusses how more could be done to teach and disseminate information on laboratory safety, suggesting that it should be embedded in chemistry courses from the very beginning so that it "becomes a part of the fabric of everyday practice rather than a lazy afterthought".

To complement this academic outlook on safety, we also spoke to Dave Laffan, a Director in Chemical Development at AstraZeneca, for an industrial perspective on the topic. In a Q\&A article in this issue, Laffan tells us about the Safety, Health and Environment committee at AstraZeneca in Macclesfield - consisting of people from across the site, from different skill groups and with various levels of seniority which has regular meetings to discuss any relevant issues. The committee also reviews 'STOP' cards, a system they use to track any situation that could have a potential impact on any aspect of health and safety. He also points out that safety considerations should not be any different when it comes to industry and academia: "the experiment doesn't know if it is an industrial or academic experiment and safety should be equally important in both environments."

Justus von Liebig famously said to August Kekulé that, "If you want to become a chemist, you will have to ruin your health." Of course, there will always be potential hazards when working in a laboratory, but nobody should be putting their health - or indeed their life - at risk for the sake of an experiment. It is the responsibility of every chemist and anybody involved in scientific research to ensure that health and safety are taken seriously and backed up with the appropriate training, monitoring, policies and procedures to minimize any possible danger.

Published online: 20 December 2019 https://doi.org/10.1038/s41557-019-0405-8 